\title{
ANÁLISE ERGONÔMICA DO REPOSITÓRIO DE OBJETOS DE APRENDIZAGEM DA ÁREA DE COMPUTAÇÃO E INFORMÁTICA - ROAI
}

\section{ERGONOMIC CRITERIA ANALYSIS OF THE LEARNING OBJECTS REPOSITORY OF INFORMATION TECHNOLOGY FIELD}

\author{
Avanilde Kemczinski ${ }^{1}$ \\ Thiago Corrêa da Silva ${ }^{1}$ \\ Kariston Pereira ${ }^{1}$ \\ Ivanete Zuchi Siple ${ }^{2}$ \\ Isabela Gasparini ${ }^{1}$
}

O objetivo deste trabalho é adequar a interface do Repositório de Objetos de Aprendizagem da área de Informática (ROAI) da UDESC/CCT/DCC aos critérios ergonômicos, base que se destina ao uso em atividades extensionistas do grupo de pesquisa. O processo de avaliação foi fundamentado em uma técnica de inspeção ergonômica via checklist (ErgoList), que tem o objetivo de avaliar a interface e a interação do sistema, baseada nos critérios ergonômicos de Scapin e Bastien. Como resultado do processo de avaliação do ROAl, foram identificadas trinta e duas não conformidades dentro dos critérios ergonômicos adotados, sendo estas classificadas de acordo com o grau de severidade juntamente com a solução do problema.

Palavras-chave: avaliação de interfaces, critérios ergonômicos, repositórios, objetos de aprendizagem.

The goal of this work is to adapt the interface of the Learning Objects Repository (LOR) of IT field (namely ROAI) from UDESC University to ergonomics criteria. ROAI is intended for use in extension activities of our research group. The evaluation process was based on an ergonomic inspection technique via checklist (Ergolist), which aims to evaluate the system' interface and interaction based on ergonomic criteria defined by Bastien and Scapin. As a result of the evaluation process of ROAl, thirty-two non-conformities were identified within the ergonomic criteria adopted, which were classified according to their degree of severity, and then we presented a solution to the problems found.

\footnotetext{
${ }^{1}$ Departamento de Ciência da Computação - Universidade do Estado de Santa Catarina avanilde@joinville.udesc.br, thiag0_c@hotmail.com, kariston@joinville.udesc.br, isabela@joinville.udesc.br

2 Departamento de Matemática - Universidade do Estado de Santa Catarina (UDESC) ivazuchi@gmail.com
} 
Keywords: evaluation of interfaces, ergonomic criteria, repositories, learning objects.

\section{Introdução}

Segundo Cybis (2010), para a escolha de uma técnica de avaliação é importante examinar as suas qualidades no confronto com os recursos disponíveis e as expectativas de resultados da avaliação. Quando se inicia um processo de avaliação de interface é importante esclarecer que, mesmo com a junção de várias técnicas e uma avaliação complexa e exaustiva, ainda existe certo grau de incerteza (BARANAUSKAS; ROCHA,2003)

A ergonomia pode ser definida como sendo "o conjunto de conhecimentos científicos relativos ao homem e necessários à concepção de instrumentos, máquinas e dispositivos que possam ser utilizados com 0 máximo de conforto, segurança e de eficácia" (WISNER, 1987).

Este artigo tem como objetivo apresentar os resultados obtidos através da avaliação ergonômica da interface do Repositório de Objetos de Aprendizagem para a Área de Informática (ROAI) da Universidade do Estado de Santa Catarina (UDESC), do Centro de Ciências Tecnológicas (CCT) do Departamento de Ciência da Computação (DCC). Neste sentido, a seção 2 define o conceito de ergonomia e interface e cita os critérios ergonômicos utilizados na avaliação da interface do ROAI, a seção 3 trata as técnicas de avaliação de interfaces e realiza a justificativa da técnica escolhida, a seção 4 define o sistema avaliado, a seção 5 apresenta o resultado da avaliação do sistema e as soluções alternativas aplicadas para que a base possa ser disponibilizada sociedade e aplicados em outros projetos de pesquisa e extensão e por fim a seção 6 contêm as conclusões do trabalho, seguida das referências bibliográficas.

\section{Ergonomia, Interface e Critérios Ergonômicos}

Segundo Montmollin (1971), a ergonomia é a tecnologia das comunicações homem-máquina. A ergonomia é uma ciência interdisciplinar, ela compreende a fisiologia e a psicologia do trabalho. O objetivo prático da ergonomia é a adaptação do posto de trabalho, dos instrumentos, das 
máquinas, dos horários, do meio ambiente às exigências do homem. A realização de tais objetivos, ao nível industrial, propicia uma facilidade do trabalho e um rendimento do esforço humano (GRANDJEAN, 1968).

Uma regra ergonômica constitui um princípio de concepção ou de avaliação cujo objetivo é obter ou garantir que a interface homem-máquina seja ergonômica. As regras ergonômicas devem respeitar critérios ergonômicos na concepção da interface. Os critérios, por sua vez, constituem uma dimensão que nos leva a uma interface elaborada, eficiente, sofisticada, mais cordial e menos sujeita a erro (SCAPIN,1993).

A interface com o usuário é o mecanismo por meio do qual se estabelece um diálogo entre o programa e o ser humano. Se os fatores humanos estiverem sido levados em conta, o diálogo será harmonioso e um ritmo será estabelecido entre o usuário e o programa.

... o objetivo final da tecnologia computacional é fazer com que - computador desapareça, que a tecnologia seja tão transparente, tão invisível ao usuário, que na prática, o computador não exista.... Isso permite que o usuário interaja com o computador como se o computador fosse outro ser humano. (ANERSON, 1989, p. 91)

Na comunicação via computador, ressalta-se a importância da interface do software como ponto fundamental na interação humano-computador. Interface, segundo Vavassori (1995), é o modo pelo qual o usuário mapeia suas tarefas sobre o conjunto de ferramentas disponíveis em um sistema computacional. Interface é toda a porção do sistema com a qual o usuário mantém contato físico (motor ou perceptivo) ou conceitual durante a interação (MORAN, 1981). A interface deve ser natural e intuitiva. O que se deseja é uma interface que minimize o treinamento necessário, permitindo que 0 usuário consiga realizar suas tarefas sem dúvidas ou perdendo tempo. No entanto, a interação do ser humano com o computador não é natural, pois o homem não nasce com a capacidade de operar os dispositivos físicos e lógicos usados na interação com os computadores. Prova disso é a dificuldade encontrada por grande parte da população para operar terminais bancários. Frente a este contexto, é necessário tratar os aspectos relacionados à "Ergonomia de Software. Schneiderman (2002) conceitua Ergonomia de 
Software como sendo "um conjunto de regras, normas e recomendações utilizadas na concepção de ferramentas através do computador colocadas a disposição do usuário".

O conjunto atual de critérios ergonômicos foi definido em 1993, por Scapin e Christian Bastien, pesquisadores do INRIA (Institut National de Recherche en Informatique et en Automatique da França). É composto de oito critérios principais, que se subdividem conforme apresentado na tabela 1:

Tabela 1 - Descrição dos Critérios Ergonômicos

\begin{tabular}{|c|c|c|}
\hline $\begin{array}{c}\text { Critério } \\
\text { Ergonômico }\end{array}$ & Descrição & Subcritérios \\
\hline \multirow{4}{*}{ Condução } & \multirow{4}{*}{$\begin{array}{l}\text { O software deve informar e } \\
\text { conduzir o usuário na interação } \\
\text { com o computador; Possibilitar } \\
\text { a localização do usuário, o } \\
\text { conhecimento das ações } \\
\text { permitidas, bem como suas } \\
\text { consequências }\end{array}$} & Convite \\
\hline & & $\begin{array}{l}\begin{array}{l}\text { Agrupamento de itens por } \\
\text { formato }\end{array} \\
\begin{array}{l}\text { Agrupamento de itens por } \\
\text { localização }\end{array} \\
\end{array}$ \\
\hline & & Legibilidade \\
\hline & & Feedback imediato \\
\hline \multirow{3}{*}{ Carga de trabalho } & \multirow{3}{*}{$\begin{array}{l}\text { Redução da carga cognitiva e } \\
\text { aumento da eficiência do } \\
\text { diálogo; Quanto menos } \\
\text { informações desnecessárias, } \\
\text { menor será a probabilidade de } \\
\text { cometer erros }\end{array}$} & Concisão \\
\hline & & Ações mínimas \\
\hline & & Densidade informacional \\
\hline \multirow[b]{2}{*}{ Controle Explícito } & \multirow{2}{*}{$\begin{array}{l}\text { Delegar o controle sobre as } \\
\text { ações efetuadas pelo sistema; } \\
\text { Os erros diminuem quando os } \\
\text { usuários } \\
\begin{array}{l}\text { explicitamente e mantêm } \\
\text { controle sobre as suas } \\
\text { entradas }\end{array}\end{array}$} & Ações explícitas do usuário \\
\hline & & Controle do usuário \\
\hline \multirow[b]{2}{*}{ Adaptabilidade } & \multirow{2}{*}{$\begin{array}{l}\text { O sistema deve reagir } \\
\text { conforme o contexto, e } \\
\text { conforme as necessidades e } \\
\text { preferências do usuário }\end{array}$} & Flexibilidade \\
\hline & & Experiência do usuário \\
\hline \multirow{3}{*}{ Gestão de Erros } & \multirow{3}{*}{$\begin{array}{l}\text { Refere-se aos mecanismos } \\
\text { que permitem evitar ou reduzir } \\
\text { os erros, e quando eles } \\
\text { ocorrem que favoreçam sua } \\
\text { correção }\end{array}$} & Proteção contra erros \\
\hline & & Mensagens de erro \\
\hline & & Correção dos erros \\
\hline $\begin{array}{l}\text { Significado dos } \\
\text { Códigos }\end{array}$ & $\begin{array}{l}\text { Adequação semântica entre o } \\
\text { objeto ou a informação } \\
\text { apresentada ou pedida, e sua } \\
\text { referência; Ex. O título deve } \\
\text { transmitir o que ele representa }\end{array}$ & \\
\hline
\end{tabular}




\begin{tabular}{|l|l|l|}
\hline Homogeneidade & $\begin{array}{l}\text { e ser distinto de outros títulos } \\
\text { A interface do sistema deve ser } \\
\text { consistente (padrões para } \\
\text { códigos, denominações, } \\
\text { formatos, procedimentos, etc...) }\end{array}$ & \\
\hline Compatibilidade & $\begin{array}{l}\text { Adequação das tarefas e } \\
\text { características do usuário, com } \\
\text { a organização das entradas, } \\
\text { saídas e diálogos da aplicação }\end{array}$ & \\
\hline
\end{tabular}

Fonte: Cybis (1993)

Estes critérios (descritos na tabela 1) são extremamente úteis para definir quais são as qualidades que devem ser atribuídas ao software durante todo o projeto (da concepção à avaliação), para que satisfaçam os usuários quanto à sua utilização.

\section{Avaliação de Interfaces}

De acordo com Cybis (2003) as técnicas de avaliação de interfaces podem ser classificadas em três grupos: a) analíticas, que buscam prever os erros de projeto de interfaces sem a participação direta de usuários, ou seja, baseadas no conhecimento/competência de um especialista; b) empíricas, que também são chamadas de objetivas ou definitivas, contam com a participação direta do usuário e buscam levantar os problemas da interface a partir da observação interagindo com o sistema; e c) prospectivas, estas buscam a opinião do usuário em relação à interação com o sistema, verificando se o usuário está satisfeito em relação ao sistema e sua operação. Na tabela 2 descrevem-se estas técnicas de avaliação.

\section{Tabela 2 - Descrição das Técnicas de Avaliação Ergonômica de Interfaces}

\begin{tabular}{|c|l|l|}
\hline \multirow{2}{*}{$\begin{array}{c}\text { Técnicas } \\
\text { Analíticas }\end{array}$} & $\begin{array}{l}\text { Avaliação } \\
\text { Heurística técnica os avaliadores julgam a } \\
\text { interface baseados em seu conhecimento. } \\
\text { Os especialistas examinam o sistema e } \\
\text { fazem um diagnóstico dos problemas e } \\
\text { barreiras que os usuários provavelmente } \\
\text { encontrarão durante a interação } \\
\text { (NIELSEN, 1994). }\end{array}$ \\
\cline { 2 - 3 } & $\begin{array}{l}\text { São utilizadas listas de verificação } \\
\text { (checklists). Neste tipo de técnica são as } \\
\text { qualidades da ferramenta (checklists) que } \\
\text { são responsáveis pela identificação dos } \\
\text { problemas existentes na interface } \\
\text { (BARROS, 2003). }\end{array}$ \\
\hline Inspeção de \\
Conformidade
\end{tabular}




\begin{tabular}{|c|c|c|}
\hline & Percurso Cognitivo & $\begin{array}{l}\text { Segundo Prates e Barbosa (2003), esta } \\
\text { técnica visa avaliar principalmente a } \\
\text { facilidade de aprendizado do sistema, em } \\
\text { particular pela exploracão dos usuários. }\end{array}$ \\
\hline & $\begin{array}{c}\text { Análise Hierárquica } \\
\text { de Tarefas }\end{array}$ & $\begin{array}{l}\text { Essa técnica visa verificar problemas na } \\
\text { interface antes mesmo que ela tenha sido } \\
\text { desenhada CYBIS (2003). }\end{array}$ \\
\hline & $\begin{array}{l}\text { Ensaios de } \\
\text { Interação }\end{array}$ & $\begin{array}{l}\text { São experimentos controlados onde } \\
\text { ocorrem simulações de uma situação real } \\
\text { de trabalho, em campo ou em laboratório, } \\
\text { com o objetivo de revelar problemas } \\
\text { ligados à utilização do sistema } \\
\text { (LUZZARDI, 2003). }\end{array}$ \\
\hline $\begin{array}{l}\text { Técnicas } \\
\text { Empíricas }\end{array}$ & $\begin{array}{c}\text { Sistemas de } \\
\text { Monitoramento }\end{array}$ & $\begin{array}{l}\text { Esta avaliação é baseada em utilitários de } \\
\text { software que permanecem residentes na } \\
\text { máquina do usuário, capturando e } \\
\text { registrando todos os aspectos das } \\
\text { interações do usuário com seu aplicativo } \\
\text { em sua própria realidade de trabalho } \\
\text { (LUZZARDI, 2003). }\end{array}$ \\
\hline \multirow{3}{*}{$\begin{array}{c}\text { Técnicas } \\
\text { Prospectivas }\end{array}$} & Questionário & $\begin{array}{l}\text { Questionários podem identificar o perfil dos } \\
\text { usuários, determinar o grau de satisfação } \\
\text { dos usuários com relação à interface ou } \\
\text { estruturar informações sobre problemas } \\
\text { identificados por usuários (WINCKLER, } \\
\text { 2001). }\end{array}$ \\
\hline & Entrevista & $\begin{array}{l}\text { As entrevistas podem ser consideradas } \\
\text { questionários interativos, } \\
\text { classificadas em dirigidas quando endo } \\
\text { efetuada a partir de um questionário } \\
\text { elaborado previamente ou informais } \\
\text { quando não existe um protocolo } \\
\text { estabelecido com questões prévias } \\
\text { (FIALHO e SANTOS, 1995 apuo } \\
\text { MEDEIROS, 1999). }\end{array}$ \\
\hline & $\begin{array}{l}\text { Retorno de } \\
\text { Opiniões do } \\
\text { Usuário }\end{array}$ & $\begin{array}{l}\text { Esta técnica consiste em coletar } \\
\text { reclamações e/ou elogios do usuário } \\
\text { através da disponibilização de "quadros de } \\
\text { avisos eletrônicos", "caixas de sugestões" } \\
\text { on-line, relatórios on-line, verbalização por } \\
\text { telefone ou relatos com caneta e papel } \\
\text { (QUEIROZ, 2006). }\end{array}$ \\
\hline
\end{tabular}

Inicialmente, pesquisou-se qual o tipo de técnica seria adequada para a avaliação da interface do ROAl, levando em consideração a necessidade da adequação da interface deste sistema aos critérios ergonômicos. O processo de avaliação foi fundamentado em uma técnica analítica de inspeção ergonômica via checklist, que tem o objetivo avaliar a interface e a interação do sistema. O que se mostrou mais adequada e sendo escolhida para a avaliação 
foi a ferramenta Ergolist, uma ferramenta baseada nos critérios ergonômicos de Scapin e Bastien (1993), pois ela é de fácil e rápida aplicação comparada a outras listas de verificação estudadas. Esta ferramenta caracteriza-se na aplicação dos critérios ergonômicos através de questões apresentadas na lista de verificação, apoiado em glossário e informações detalhadas sobre o critério que está sendo avaliado. $O$ avaliador assinala as respostas que na sua análise são as mais adequadas naquela situação e o sistema armazena as respostas. No término do processo, o sistema gera um laudo final sobre as questões que apresentaram conformidade ergonômica, as que não são aplicáveis e as que não apresentam conformidade ergonômica.

A seguir apresenta-se o sistema avaliado, bem como as não conformidades encontradas na interface do ROAI de acordo com os critérios ergonômicos.

\section{Repositório de Objetos de Aprendizagem para a Área de Informática} (ROAl)

O repositório tem como objetivo a disseminação de conteúdos educacionais, através de um ambiente específico para catalogação e inclusão de OAs (Objetos de Aprendizagem) para a área de informática e computação. Para tanto, o sistema possui três visões de usuário distintas:

- Visitante: se não estiverem autenticados no repositório, os usuários são considerados "usuários visitantes". Possuem privilégios, tais como pesquisar, visualizar e salvar objetos de aprendizagem.

- Participante: uma vez autenticados, os usuários possuem privilégios para submeterem objetos, além de pesquisar, visualizar, avaliar e salvar os objetos.

- Administrador: possui pleno acesso a todas as funções em todos os itens gerenciais do repositório.

O ROAl é um repositório temático e local, pois os objetos armazenados nele abordam conteúdos da área de informática e o seu planejamento, desenvolvimento e implantação estão sendo realizados na Universidade do Estado de Santa Catarina, UDESC. 
A figura 1 a seguir apresenta a página inicial do sistema.

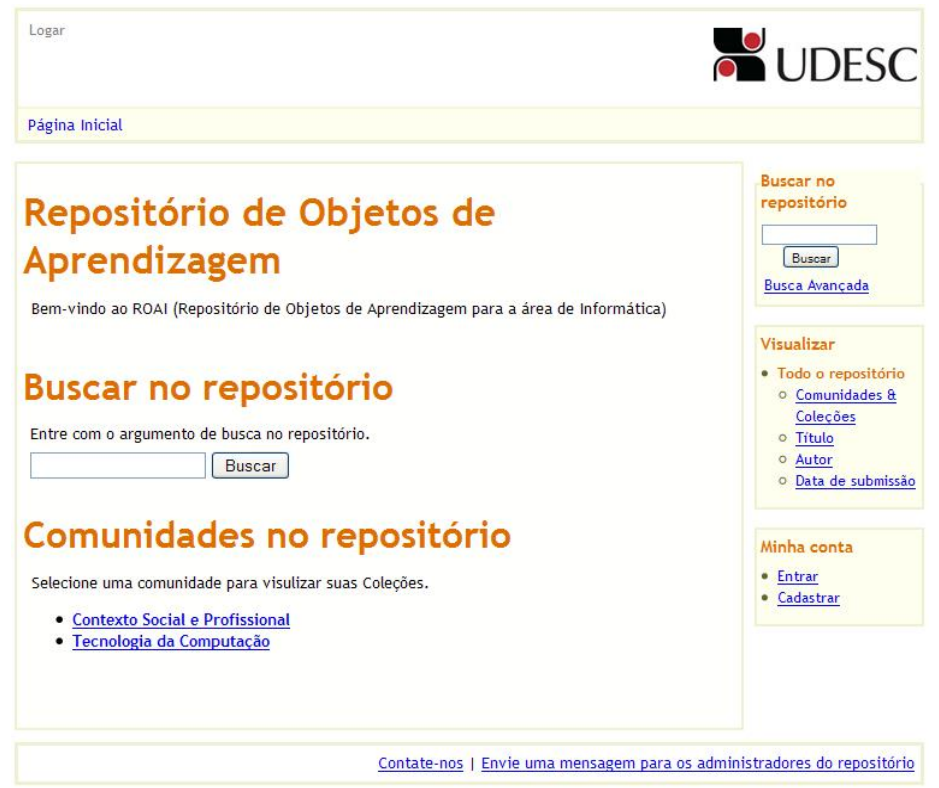

Figura 1- Página inicial do ROAI

A página inicial do repositório tem como destaque o campo de busca para pesquisa dos OAs e links para as comunidades existentes. O campo de busca realiza a pesquisa em todas as comunidades e coleções. O menu ao lado direito é constante em todas as páginas do sistema, exceto na visão dos administradores do repositório, onde é acrescido itens gerenciais. Este menu contém os links para navegação e visualização dos OAs através das comunidades e coleções, além da navegação por índices de autores, por título de OAs e pela data de submissão dos objetos no ROAI.

\section{Avaliação da Interface do ROAl}

Para realizar a avaliação por meio da Inspeção de Conformidade foi utilizada a ferramenta ErgoList, como já justificado anteriormente na seção 3. Foi seguida a seguinte metodologia:

1.Estudo sobre ergonomia e foco nos critérios ergonômicos.

2.Análise das técnicas de avaliação de interfaces para escolher a que melhor se adequa, com foco na ferramenta escolhida para a tarefa. 
3.Navegação pelo sistema, dando atenção a todos os detalhes e realizando as tarefas disponíveis aos usuários;

4.Resposta às questões do ErgoList, sempre atentando para as informações no glossário e na opção "Mais sobre..." e recorrendo ao sistema em casos de dúvidas;

5.Revisão da avaliação por outro especialista.

Das 194 questões propostas pelo ErgoList, 115 obtiveram resposta afirmativa (a interface atendeu os critérios ergonômicos), 32 obtiveram resposta negativa (a interface não atendeu os critérios ergonômicos) e 47 não puderam ser aplicadas ao ROAI, conforme pode ser observado no Gráfico 1.

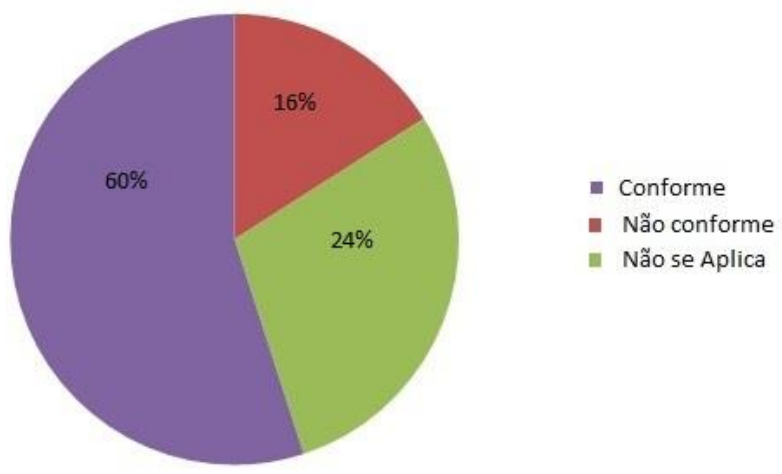

\section{Gráfico 1. Resultado geral da aplicação do ErgoList}

As 194 questões do ErgoList são divididas em 18 checklists específicos para cada um dos critérios elementares propostos por Scapin e Bastien (1993), sendo que o Gráfico 2 apresenta os resultados obtidos para cada um desses critérios. 


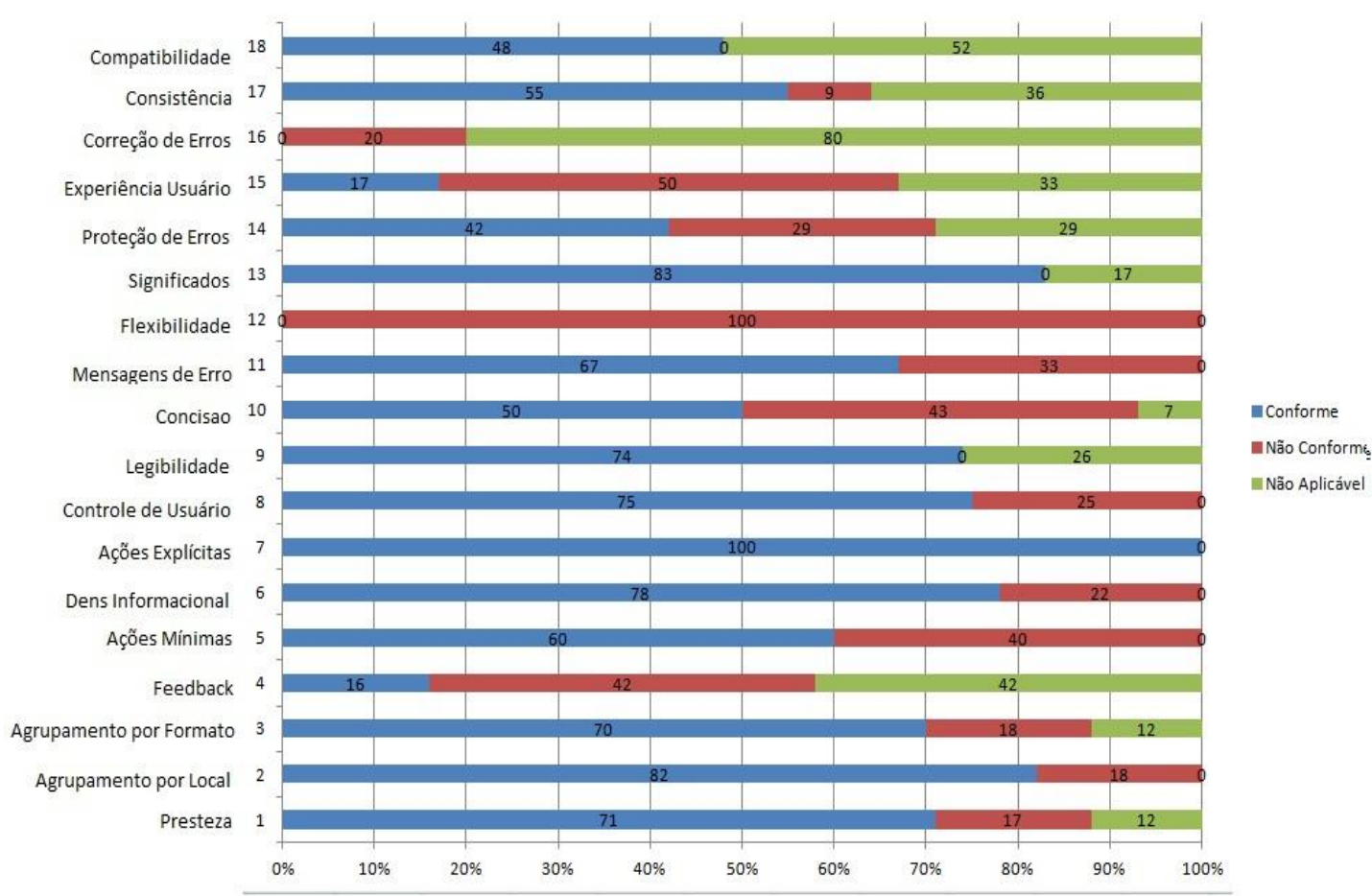

Gráfico 2. Resultados da aplicação do ErgoList por critérios elementares

Observando o gráfico 2 percebe-se que os critérios Flexibilidade e Correção de Erros foram os que obtiveram os piores resultados. Todos os itens do checklist sobre Flexibilidade não estavam conforme, e apenas $20 \%$ dos itens do checklist sobre Correção de Erros estavam conforme. Os critérios Compatibilidade, Significados, Legibilidade e Ações Explícitas foram os que obtiveram os melhores resultados atendendo a $100 \%$ dos critérios relacionados que puderam ser aplicados ao ROAI.

\subsection{Classificação das não conformidades encontradas}

Segundo Cybis (2010), a análise da natureza de um problema de interface permite classificá-lo como: a) Barreira - quando se refere a um aspecto da interface no qual o usuário esbarra sucessivas vezes e não aprende a suplantá-lo e acaba fazendo com que ele desista de usar uma função do sistema; b) Obstáculo - quando o usuário esbarra no aspecto, mas aprende a suplantá-lo. A realização dessa tarefa se dará sempre na perda de desempenho; c) Ruído - quando um aspecto da interface causa uma diminuição de seu desempenho na tarefa. 
Em relação ao tipo de tarefa na qual o problema de usabilidade se manifesta, ele pode ser classificado como (CYBIS, 2003): i) Principal - no caso de um aspecto da interface que compromete a realização de tarefas frequentes ou importantes; ii) Secundário - no caso de tarefas pouco frequentes ou pouco importantes.

E em relação ao tipo de usuário que o problema de usabilidade afeta, ele pode ser classificado como (CYBIS, 2003): 1) Geral - quando afeta função de todos os usuários; 2) Inicial - no caso de atrapalhar o usuário novato ou intermitente durante a realização de sua tarefa; 3) Avançado - no caso de um aspecto da interface que atrapalha o usuário especialista durante a realização de sua tarefa e 4) Falso - no caso de não trazer qualquer prejuízo ao usuário, nem à sua tarefa, tratando-se de um engano provocado pela falta de experiência do avaliador ou de uma deficiência em sua ferramenta de avaliação.

De acordo com estas classificações a tabela 3 determina o grau de severidade dos problemas, estabelecendo uma escala de 4 a 0 (NIELSEN, 1994), sendo: 4 -imperativa a correção; 3 - alta prioridade de correção; 2 baixa prioridade de correção; 1 - problema estético; 0 - não é considerado necessariamente como um problema.

Tabela 3 - Classificação das prioridades de correção das não conformidades (AMARAL, 2006).

\begin{tabular}{|l|l|l|l|}
\hline NATUREZA & TAREFA & USUÁRIO & PRIORIDADE \\
\hline Barreira & Principal & Geral & 4 \\
\hline Barreira & Principal & Inicial & 4 \\
\hline Barreira & Principal & Avançado & 4 \\
\hline Barreira & Secundária & Geral & 4 \\
\hline Barreira & Secundária & Inicial & 3 \\
\hline Barreira & Secundária & Avançado & 3 \\
\hline Obstáculo & Principal & Geral & 4 \\
\hline Obstáculo & Principal & Inicial & 3 \\
\hline Obstáculo & Principal & Avançado & 3 \\
\hline Obstáculo & Secundária & Geral & 3 \\
\hline Obstáculo & Secundária & Inicial & 2 \\
\hline Obstáculo & Secundária & Avançado & 2 \\
\hline Ruído & Principal & Geral & 2 \\
\hline Ruído & Principal & Inicial & 2 \\
\hline Ruído & Principal & Avançado & 2 \\
\hline Ruído & Secundária & Geral & 2 \\
\hline Ruído & Secundária & Inicial & 1 \\
\hline Ruído & Secundária & Avançado & 1 \\
\hline
\end{tabular}


A análise para identificar as não conformidades da interface do ROAl, aplicando o Ergolist resultou em 32 não conformidades. Essas não conformidades, de acordo com a tabela 3, foram priorizadas de 4 a 0 (sendo 4 as mais urgentes e 0 não havendo necessidade de correção), conforme pode ser observado na Tabela 4.

Tabela 4. Não conformidades identificadas e prioridades de correção

\begin{tabular}{|l|c|}
\cline { 2 - 2 } \multicolumn{1}{c|}{} & $\begin{array}{l}\text { Quantidade de } \\
\text { Problemas }\end{array}$ \\
\hline Prioridade 4 & 5 \\
\hline Prioridade 3 & 0 \\
\hline Prioridade 2 & 16 \\
\hline Prioridade 1 & 5 \\
\hline Prioridade 0 & 6 \\
\hline Total & 32 \\
\hline
\end{tabular}

Observando a

Tabela 4 vê-se que para a prioridade 1 e 4, foram identificadas 5 (16\%) não conformidades. Nota-se também que metade (50\%) das não conformidades identificadas pelo ErgoList foram classificadas na prioridade 2, não foram identificadas não conformidades com prioridade 3 , isto de um total de 32 não conformidades encontradas.

\subsection{Solução para as não conformidades}

As não conformidades de prioridade 4 são eventos que impossibilitam 0 usuário de realizar alguma tarefa no sistema, estas puderam ser corrigidas adicionando, ao lado do local das principais tarefas, orientações para o usuário por meio de um botão de ajuda que, quando acionado exibe textos explicativos para a realização das tarefas ou solução do problema. Outra necessidade de correção está relacionada com a tradução completa do sistema para a língua portuguesa. As demais não conformidades, estão classificadas com prioridade 2, puderam ser adequadas com algumas alternativas como: criar botões padrões para todas as ações (funcionalidades do sistema), diferenciar ações destrutivas como exclusão de OAs; adicionar mensagem de feedback para 
todas as ações do sistema, por exemplo, uma mensagem de feedback confirmando ou não a submissão ou avaliação de um OA; exibir informação de tempo de processamento nas funções de submissão e avaliação do objeto; incluir opção de interromper um processo pelo usuário; incluir teclas para acelerar a navegação entre as páginas do sistema.

As não conformidades de nível 1, que tratam de problemas estéticos foram prescritas: colocar um comentário ou alerta sobre alterações feitas no perfil do usuário, na distribuição de novas tarefas (avaliação de $O A$ ), na exclusão de uma coleção, uma comunidade ou um usuário cadastrado; exibir uma mensagem de alerta quando de uma ação significativa; padronizar e diferenciar as mensagens por tipo de usuário, por exemplo, adicionar uma opção "ver mais" para usuário iniciante e mensagens do tipo: "deseja realmente sair desta sessão? Haverá perda dados nesta ação". Dessa forma, espera-se obter uma nova interface para o ROAl que garanta a ergonomia para o usuário.

\section{Conclusão}

Através da aplicação do ErgoList foi observado que a interface do ROAI apresenta inúmeras não conformidades, de acordo com os critérios ergonômicos de Scapin e Bastien (1993). No entanto, no geral, o resultado da avaliação via inspeção foi satisfatório, visto que somente $32 \%$ dos elementos da interface do sistema apresentaram problemas. Pode ser observado também que as não conformidades identificadas não são itens de difícil solução, enquanto os itens de conformidade identificados são importantes em sistemas web, pois tornam o ambiente agradável, fácil de aprender e fácil de usar.

Como trabalho futuro novas avaliações deverão ser realizadas para identificar as não conformidades com base nos critérios de usabilidade e de acessibilidade. A partir das não conformidades encontradas na interface do sistema, através da avaliação dos critérios ergonômicos e posteriormente de usabilidade e acessibilidade, uma nova interface será projetada Em paralelo a este projeto, foi adicionado ao ROAI a busca semântica no repositório e o desenvolvimento de uma metodologia e ferramenta para a construção de OAs interativos para o usuário final, que também visam garantir uma melhora na 
eficiência do uso, bem como a satisfação do usuário, critérios importantes na qualidade de uso do sistema.

Finalmente, a expectativa é tornar o repositório de uso publico e que possa atender a projetos de pesquisa e extensão do grupo de Pesquisa que o desenvolveu.

\section{REFERÊNCIAS}

AMARAL, D. Adequação da Interface do SAI-AE aos Critérios Ergonômicos e de Usabilidade. 2006. Trabalho de Conclusão de Curso. (Graduação em Bacharelado em Ciência da Computação) - Universidade do Estado de Santa Catarina. Orientador: Avanilde Kemczinski.

ANDERSON, J. J. Multimedia: about interface. MacUser, v. 5, n. 3, p. 89-96, Mar 1989.

BARANAUSKAS, M. C. C.; ROCHA, H. V. Design e Avaliação de Interfaces Humano Computador. 2003: Unicamp/Nied. São Paulo

BARROS, V. T. O. Avaliação da Interface de um Aplicativo Computacional Através de Teste de Usabilidade, Questionário Ergonômico e Análise Gráfica do Design. 2003 Dissertação (Mestrado em Engenharia de Produção) Programa de Pós-Graduação em Engenharia de Produção - Universidade Federal de Santa Catarina, Florianópolis.

CYBIS, W; BETIOL, A. H.; FAUST, R. Ergonomia e Usabilidade: conhecimentos, métodos e aplicações. Novatec, 2a. edição, 2010.

CYBIS, W. A. Engenharia de Usabilidade: uma abordagem ergonômica. 2003. Apostila para o curso de Pós-Graduação em Engenharia de Produção Laboratório de Utilizabilidade de Informática - Universidade de Santa Catarina: Florianópolis.

ErgoList. (1998) "Projeto ErgoList". Home Page do Projeto. Disponível em: <http://www.labiutil.inf.ufsc.br/ergolist/>. Acesso em: 25 maio 2006. 
FERLIN, J. Repositório de objetos de aprendizagem para a área de informática. Universidade do Estado de Santa Catarina, 2009. Trabalho de Conclusão de Curso. - Universidade do Estado de Santa Catarina. Orientador: Avanilde Kemczinski.

FIALHO, F.; SANTOS, N. Manual de Análise Ergonômica do Trabalho. Curitiba: Genesis, 1995.

GRANDJEAN, E. 1968. Fatigue: Its physiological and psychological significance.Acesso em 12 jun. 2008. http://www.ergonomia.com.br/

LIMA, S. L. S. Ergonomia Cognitiva e a Interação Pessoa-Computador: análise da usabilidade da urna eletrônica 2002 e do módulo impressor externo. Dissertação (Mestrado em Engenharia de Produção) - Programa de PósGraduação em Engenharia de Produção - Universidade Federal de Santa Catarina, Florianópolis, 2003.

LUZZARDI, P. R. G. Critérios de Avaliação de Técnicas de Visualização de Informações Hierárquicas. Tese (Doutorado em Ciência da Computação) Programa de Pós-Graduação em Computação - Universidade Federal do Rio Grande do Sul, Porto Alegre, 2003.

MOÇO, S. S., O uso de cenários como uma técnica de apoio para avaliações ergonômicas de softwares interativos.1996. Dissertação (Mestrado em Engenharia de Produção) - Programa de Pós Graduação em Engenharia de Produção, Universidade Federal de Santa Catarina, Florianópolis.

MONTMOLLIN, M. L’Ergonomie. Paris: La Découverte, 1971.

MORAN, T. P. The command language grammar: A representation for the user interface of interactive computer systems. International Journal of ManMachine Studies, 1981, 15, 1, 3-50. 
NIELSEN, J. (1994). Enhancing the explanatory power of usability heuristics. In;- Proceedings of ACM CHI94 Conference of human Factors in Computing Systems. Monterey, CA: ACM., 1994.

QUEIROZ, J. E. R. Abordagem Híbrida para a Avaliação da Usabilidade de Interfaces com o Usuário. Projeto de Pesquisa da Universidade Federal de Campina Grande, 2006.

SCAPIN, D. L.; BASTIEN, J. M. C. Ergonomic Criteria for the Evaluation of Human-Computer Interfaces. Relatório Técnico № 156 - Institut National de Recherche en Informatique et en Automatique (INRIA), França, 1993. Disponivel online: http://hal.inria.fr/docs/00/07/00/12/PDF/RT-0156.pdf. Acessado em maio de 2012.

SHNEIDERMAN, B. The Designing the User Interface, Addison-Wesley, 2002.

VAVASSORI, F. Método Heurístico para Avaliação e Projeto de Interfaces Homem-Computador . 1995. Monografia Bacharelado em Ciência da Computação). UCPEL, Pelotas.

WINCKLER, M. A. A. Avaliação de Usabilidade de Sites Web. Workshop sobre Fatores Humanos em Sistemas Computacionais, Florianópolis, 2001.

WISNER, A. Por Dentro do Trabalho: ergonomia: método \& técnica. São Paulo: FTD: Oboré, 1987. 